\title{
Lung Biphasic Synovial Sarcoma
}

National Cancer Institute

\section{Source}

National Cancer Institute. Lung Biphasic Synovial Sarcoma. NCI Thesaurus. Code C45632.

A biphasic synovial sarcoma arising from the lungs. 